\title{
Role of cyclin-dependent kinase 5 in early brain injury following experimental subarachnoid hemorrhage
}

\author{
YU DING, LIEXIANG ZHANG, WEI ZHOU, HAI LU, XINGDE GAO, \\ JIAN LI, JINGDE LIU, XIAOWANG NIU and JING ZHENG
}

\begin{abstract}
Department of Neurosurgery, The Affiliated Suqian Hospital of Xuzhou Medical University, Suqian, Jiangsu 223800, P.R. China
\end{abstract}

Received March 5, 2020; Accepted September 9, 2021

DOI: $10.3892 /$ etm.2021.11070

\begin{abstract}
Increasing evidence indicates that early brain injury (EBI) can contribute to poor outcomes following subarachnoid hemorrhage $(\mathrm{SAH})$, and is associated with apoptosis. Cyclin-dependent kinase $5(\mathrm{Cdk} 5)$ is a key mediator of neuronal viability. The role of Cdk5 in several neurological disorders has been elucidated; however, its role in EBI after $\mathrm{SAH}$ remains unclear. The present study aimed to explore the involvement of Cdk5 in EBI after SAH. The expression levels of Cdk5, Cdk5 phosphorylated at Tyr15 (Cdk5-pTyr15) and p25 (a Cdk5 activator) were assessed by western blotting, and the cell distribution of $\mathrm{Cdk} 5$ was demonstrated by double immunofluorescence. The expression levels of caspase-3 and cytochrome c were evaluated by western blotting to assess the severity of neuronal apoptosis. Nissl and TUNEL staining experiments were performed to observe the effects of roscovitine, a Cdk5 inhibitor, on EBI following SAH. The results indicated that the expression levels of Cdk5, p25 and Cdk5-pTyr15 significantly increased in the rat temporal cortex following SAH. Immunofluorescence staining indicated that Cdk5 was expressed in the neurons and astrocytes of the rat cortex after $\mathrm{SAH}$ and that $\mathrm{Cdk} 5$ underwent nuclear translocation in neurons. Roscovitine administration effectively inhibited Cdk5 activation. In conclusion, roscovitine treatment significantly mitigated EBI and alleviated cerebral edema following SAH. These findings suggest that Cdk5 is an important target in SAH therapy.
\end{abstract}

\section{Introduction}

Subarachnoid hemorrhage (SAH) is frequently observed in severe diseases, and is associated with a high mortality ratio

Correspondence to: Dr Jing Zheng, Department of Neurosurgery, The Affiliated Suqian Hospital of Xuzhou Medical University, 138 Huanghe South Road, Suqian, Jiangsu 223800, P.R. China E-mail: dr_zhengjing@163.com

Key words: cyclin-dependent kinase 5, subarachnoid hemorrhage, apoptosis, early brain injury, brain edema and high disability rate worldwide (1). The mortality rate from SAH is $~ 50 \%$ in population-based studies in the USA with a trend towards gradual improvement $(2,3)$. The majority of SAH-related studies performed previously focused on cerebral vasospasm (CVS) following SAH. Several treatments, such as circulatory volume expansion, endothelin receptor antagonists and calcium antagonists, have been applied to prevent vasospasm; however, the reversal of CVS did not improve clinical outcomes following SAH (4).

Increasing numbers of clinical studies have reported that the majority of post-SAH mortalities occur rapidly and are caused largely by early brain injury (EBI), which begins to develop within minutes after the initial bleeding started (5-7). Mounting evidence shows that the alleviation of EBI increases neurological function and ameliorates cognitive deficits in experimental SAH $(8,9)$. Therefore, EBI, which occurs within 24-72 $\mathrm{h}$ following SAH, may account for the abovementioned poor outcomes following SAH.

The molecular mechanism underlying EBI development after SAH is complex and involves cerebral edema, blood-brain barrier (BBB) disruption or microvasculature dysfunction. Among the mechanisms aforementioned, apoptosis has been revealed to be an important player in EBI (10). Endothelial apoptosis has been revealed to cause BBB breakdown and consequently induce brain edema. Moreover, a study also revealed that neuronal apoptosis is an essential part of EBI following SAH (11), whereas inhibition of apoptosis has been demonstrated to exert protective effects against SAH, thereby decreasing mortality and improving neurological outcomes in SAH animal models (12).

Cyclin-dependent kinase $5(\mathrm{Cdk} 5)$, a proline-directed serine-threonine kinase, is highly expressed in cells of the nervous system (13). A previous study has demonstrated that Cdk5 is involved in balancing survival and apoptosis of neurons in the central nervous system (14). In contrast to canonical Cdk-signaling, Cdk5 does not function in cell cycle regulation; Cdk5 is instead involved in cytoskeletal protein phosphorylation, neurogenesis, cognition and neuronal survival in the brain under physiological conditions (15). Cdk5 is also activated by the non-cyclin proteins p35 and p39, which are predominantly expressed in the CNS under physiological conditions (16). However, during neurotoxic stress, Cdk5 binds with p25 (a Cdk5 activator), which triggers Cdk5 hyperactivation. 
Aberrant Cdk5 activity leads to the hyperphosphorylation of several downstream substrates, contributing to cellular dysfunction and the development of neurological disease (17).

Previous studies have indicated that expression levels of Cdk5 and Cdk5 phosphorylated at Tyr15 (Cdk5-pTyr15) in the human brain increased significantly after acute ischemic stroke, and that inhibition of Cdk5 significantly reduced infarct volumes on day 1 in animal stroke models (18-20). However, the biological functions of $\mathrm{Cdk} 5$ in EBI after $\mathrm{SAH}$ are still unclear. Thus, the present study aimed to investigate the role of Cdk5 in EBI following SAH.

\section{Materials and methods}

Animal sourcing. In total, 138 male Sprague-Dawley rats (weight, 250-320 g; 6-8 weeks) were purchased from Jinling Hospital (Nanjing, China). Rats were first acclimated to animal cabinets at $23 \pm 1^{\circ} \mathrm{C}$, with $100 \%$ fresh air, $50 \%$ humidity and free access to food and water under 12-h light/dark cycles. All study protocols regarding surgical procedures and animal usage were approved by The Ethics Committee of the Affiliated Suqian Hospital of Xuzhou Medical University (Suqian, China) and conformed to the Guide for the Care and Use of Laboratory Animals (8th edition) by National Research Council (US) Committee (21).

Induction of experimental SAH. The prechiasmatic injection model of SAH was prepared by modifying some previous procedures (22-24). Briefly, male Sprague-Dawley rats were anesthetized via intraperitoneal injections of sodium pentobarbital $(40 \mathrm{mg} / \mathrm{kg})$. A midline scalp incision was made and a 1-mm hole was created at a location 7.5-8.0 $\mathrm{mm}$ above the bregma (25). A pre-chiasmatic cistern SAH injection model (25) was established by drawing $300 \mu \mathrm{l}$ fresh autologous arterial blood from the rat's femoral artery using insulin needles before drilling a 1-mm hole above the bregma. The blood was slowly injected into the pre-chiasmatic cistern via the hole, which was fed into the hole and then removed after blood was injected. After these steps, the rats were returned to their home cages and fed normally. The room temperature was set at $23 \pm 1^{\circ} \mathrm{C}$.

Experimental design. To analyze the expression levels of Cdk5, Cdk5-pTyr15 and p25 after SAH, rats were randomly assigned into six groups in time course experiments: Sham $(\mathrm{n}=6), 6 \mathrm{~h}$ after SAH $(\mathrm{n}=6), 12 \mathrm{~h}$ after SAH $(\mathrm{n}=6), 1$ day after SAH $(n=6), 3$ days after SAH $(n=6)$ and 5 days after SAH $(n=6)$. Immunofluorescence assay was used to determine the cellular localization of Cdk5 (Sham, $n=6 ; 1$ day after $\mathrm{SAH}$, $\mathrm{n}=6$ ). The rats in the sham group were injected with the same volume of $0.9 \%$ saline into the bregma hole with reference to procedures mentioned above.

To explore the role of $\mathrm{Cdk} 5$ following $\mathrm{SAH}$, a total of 90 rats were randomly assigned to four groups: i) A sham (20\% PBS) group $(n=24)$; ii) a SAH + vehicle group $(n=24)$; iii) a $\mathrm{SAH}+$ roscovitine $(50 \mu \mathrm{g})$ group $(\mathrm{n}=18)$; and iv) a $\mathrm{SAH}+$ roscovitine $(100 \mu \mathrm{g})$ group $(\mathrm{n}=24)$. Roscovitine was administered at $30 \mathrm{~min}$ after blood injection in the $\mathrm{SAH}+$ roscovitine groups. The same volume of $20 \%$ physiological salt solution was simultaneously administered to the $\mathrm{SAH}+$ vehicle group.
All rats were sacrificed on day 1 after SAH after injection with intraperitoneal injection of $1 \%$ sodium pentobarbital $(150 \mathrm{mg} / \mathrm{kg})$. Confirmation of rat death was confirmed when the heart stopped beating. Next, the rats were transcardially perfused with PBS via the left ventricle. After the blood clots were cleared from the brain tissues, the temporal lobe was dissected for further assays. The brain tissue samples (40 $\mu \mathrm{m})$ reserved for western blotting were immediately frozen in $-80^{\circ} \mathrm{C}$ liquid nitrogen, whereas those for the TUNEL and Nissl staining experiments were fixed with $10 \%$ neutral-buffered formalin at $30^{\circ} \mathrm{C}$ for $24 \mathrm{~h}$.

Drug administration. Roscovitine, a Cdk5 inhibitor purchased from Santa Cruz Biotechnology, Inc., was dissolved in $20 \%$ dimethylsulfoxide (physiological salt solution). The induction of anesthesia was performed in a small induction chamber, where the flow of oxygen- $4 \%$ isoflurane is $21 / \mathrm{min}$ for 3-4.5 min. After $3 \mathrm{~min}$, the foot was gently pinched with tweezers at 30-sec intervals to test the anesthesia state. Roscovitine (50 or $100 \mu \mathrm{g}$ in $10 \mu \mathrm{l}$ of $20 \%$ physiological salt solution) or the same volume of physiological salt solution was intracerebroventricularly administered into each rat at $30 \mathrm{~min}$ after sham injury or SAH during, which a new $1.0-\mathrm{mm}$ lateral hole at $1.5 \mathrm{~mm}$ behind the bregma was made, through which the drug was administered. The drug dose utilized in this study was selected based on a previous study using a traumatic brain injury model (26).

Western blotting. Brain tissues isolated from the temporal cortex were homogenized. The samples were subsequently diluted (1:1) in RIPA buffer (50 mM Tris-HCl pH 7.2, $150 \mathrm{mM}$ $\mathrm{NaCl}, 1 \%$ NP-40, 0.1\% SDS, 0.5\% DOC, 1 mM PMSF, $25 \mathrm{mM}$ $\mathrm{MgCl}_{2}$ and supplemented with a phosphatase inhibitor cocktail) and boiled for $\geq 5 \mathrm{~min}$ at $100^{\circ} \mathrm{C}$. Protein concentration was estimated using the Bradford method (27). In total, $20 \mu \mathrm{g}$ proteins were then separated by $10 \%$ SDS-PAGE and transferred onto a polyvinylidene fluoride membrane, which was blocked for $30 \mathrm{~min}$ in 5\% non-fat milk in $1 \mathrm{X}$ TBS-0.1\% Tween at $37^{\circ} \mathrm{C}$ and then incubated with primary antibodies at $37^{\circ} \mathrm{C}$ for Cdk5 (1:1,000, cat. no. ab40773; Abcam), Cdk5-pTyr15 (1:1,000; cat. no. AP55874PU-N; OriGene Technologies, Inc.), p25 (1:1,000; cat. no. ab125653; Abcam) and $\beta$-actin $(1: 1,000$; cat. no. ab8226; Abcam) overnight. The membranes were then incubated with secondary antibodies [1:1,000; goat anti-rabbit IgG H\&L (HRP); cat. no. ab97051; Abcam] for $1 \mathrm{~h}$ at $37^{\circ} \mathrm{C}$ and bands of blotted protein were visualized using enhanced chemiluminescence (Thermo Fisher Scientific, Inc.). All data was normalized to the corresponding expression level of $\beta$-actin using ImageJ 1.8.0 (National Institutes of Health).

Immunofluorescence staining. Double immunofluorescence staining experiments were performed as per methods previously described by our laboratory (22). Before immunofluorescence staining, frozen temporal cortex tissue sections $(6-\mu \mathrm{m})$ were warmed at $26^{\circ} \mathrm{C}$ for $30 \mathrm{~min}$ and fixed in ice-cold acetone or other alternate $4 \%$ paraformaldehyde fixatives for $10 \mathrm{~min}$. The sections were blocked in 5\% FBS for $60 \mathrm{~min}$ and incubated with primary antibodies (Anti-NeuN antibody-Neuronal Marker; 1:100; cat. no. ab177487; Abcam; Anti-GFAP antibody; 1:100; cat. no. ab7260; Abcam; and Cdk5-antibody; 1:50; 
cat no sc-6247; Santa Cruz Biotechnology, Inc.) for $1 \mathrm{~h}$ at room temperature. The sections were washed and incubated with appropriate secondary antibodies (Alexa Fluor ${ }^{\circledR}$ 594-conjugated goat anti-rabbit IgG H\&L; 1:300, cat. no. ab150080; Abcam; and Alexa Fluor ${ }^{\circledR} 488$-conjugated goat anti-mouse IgG H\&L; 1:300, cat. no. ab150113; Abcam) for $1 \mathrm{~h}$ at $26^{\circ} \mathrm{C}$. Cell nuclei were stained using 4-diamidino-2-phenylindole (DAPI). Fluorescence microscopy was performed with a ZEISS HB050 inverted microscope (magnification, $\mathrm{x} 40$; Carl Zeiss AG) and six views of fields were processed using Image-Pro Plus 7.0 (Media Cybernetics, Inc.).

Brain water content. After the rats were sacrificed, the cerebella were removed and brain hemispheres were dissected to determine the wet weights. Brain water content was determined using the following formula [(wet weight-dry weight)/wet weight] $x 100 \%$. The brains were fixed using $4 \%$ paraformaldehyde for $30 \mathrm{~min}$ at room temperature.

Nissl staining. The same portion of the temporal cortex was used from each rat for Nissl staining. After deparaffinization and rehydration, the slice was wash in $100 \%$ ethanol twice for 10 min each, then twice $95 \%$ in ethanol for 10 min each. It was then washed in deionized $\mathrm{H}_{2} \mathrm{O}$ for $1 \mathrm{~min}$ with stirring. The slides were placed in a container and covered with $10 \mathrm{mM}$ sodium citrate buffer, $\mathrm{pH} 6.0$; or with $50 \mathrm{mM}$ glycine- $\mathrm{HCl}$ buffer (glycine: sc-29096), pH 3.5, with 0.01\% (w/v) EDTA (EDTA: sc-29092) and heated at $95^{\circ} \mathrm{C}$ for $10 \mathrm{~min}$. The $6-\mu \mathrm{m}$ sections were stained with $1 \%$ toluidine blue for $2-3 \mathrm{~min}$ at room temperature. The slices were rinsed with distilled water, and washed with 70,95 and 100\% ethanol for differentiation. Subsequently, the sections were dehydrated in an ascending xylene series and mounted using a light microscope (Leica DM750M; Leica Microsystems GmbH (x400 magnification) in six randomly selected sections were subsequently evaluated using a light microscope and surviving neurons in each section were averaged. The average number of surviving neurons across three sections was considered the number of surviving neurons for each sample. All analyses were performed by three pathologists with no knowledge of group assignments.

TUNEL staining. Apoptotic cells were detected using a TUNEL detection kit (QIA 33; Merck \& Co., Inc.) using the protocol previously described (28). Briefly, after washing with PBS, the proteinase K (20 g/ml, Sigma-Aldrich; Merck KGaA)-digested tissues were incubated in $3 \%$ hydrogen peroxid-PBS, following which they were incubated with TUNEL reaction fluid (Roche Diagnostics $\mathrm{GmbH}$ ) for $1 \mathrm{~h}$ at $37^{\circ} \mathrm{C}$. The sections were then incubated with HRP-conjugated anti-digoxigenin antibodies (1:10,000; cat. no. ab6212; Abcam) for $2 \mathrm{~h}$ at room temperature. In total, $0.05 \% \mathrm{DAB}$ was adopted to stain the sections for 5-10 min at room temperature, which were lightly counterstained with hematoxylin for $2 \mathrm{~min}$ at room temperature. Apoptotic cells were analyzed in six fields of view (magnification, x40) using a light microscope (Leica DM750M; Leica Microsystems $\mathrm{GmbH}$ ) and were processed using Image-Pro Plus 7.0 (Media Cybernetics, Inc.).

Neurological scores. Neurological scores were evaluated on day 1 after SAH using the scoring method proposed by Garcia (29).
The scoring system includes categories for spontaneous activity (0-3), symmetry of movements (0-3), symmetry of the forelimbs (0-3) and the climbing ability on the cage wall (1-3), as well as sensory scores, which were determined by assessing responses to touching of the vibrissae or the sides of the trunk (1-3). All six individual scores were summed, and the value obtained was considered the neurological score for each rat.

Statistical analysis. Data are presented as means \pm SEM and were analyzed with SPSS 17.0 (SPSS, Inc.). All the data apart from neurological score were compared using one-way ANOVA with post hoc Tukey's test. Neurological score was analyzed using Kruskal-Wallis with post hoc Dunn's Test. $\mathrm{P}<0.05$ was considered to indicate a statistically significant difference.

\section{Results}

Western blotting results of Cdk5,Cdk5-pTyr15 and p25 expression. The sham group exhibited low expression levels of Cdk5 and Cdk5-pTyr15 (Fig. 1A-C). However, following SAH, Cdk5 and Cdk5-pTyr15 expression level were significantly upregulated in the SAH group compared with those in the sham group. The expression of Cdk5 peaked on day 1, whereas Cdk5-pTyr15 expression peaked at $12 \mathrm{~h}$ following SAH ( $\mathrm{P}<0.01$; Fig. $1 \mathrm{~B}$ and $\mathrm{C})$. The time course of $\mathrm{p} 25$ expression was similar to those of Cdk5 and Cdk5-pTyr15, where p25 protein levels increased significantly and peaked on day 1 after SAH $(\mathrm{P}<0.01$; Fig. 1D) in SAH group compared with those in the sham group. The protein ratio of Cdk5-pTyr15/Cdk5 expression is presented in Fig. 1E. Following SAH, the protein ratio of Cdk5-pTyr15/Cdk5 expression were significantly regulated in the $\mathrm{SAH}$ group compared with those in the sham group, which peaked at $12 \mathrm{~h}$ following $\mathrm{SAH}(\mathrm{P}<0.01$; Fig. $1 \mathrm{E})$.

Immunofluorescence staining of Cdk5 after SAH. Cdk5 was colocalized with neuronal nuclei in the sham and $\mathrm{SAH}$ groups, suggesting that Cdk5 is expressed in both healthy and post-SAH rat brain neurons. Following SAH, Cdk5 translocated to the nucleus in the SAH group but not in the sham group (Fig. 2). Cdk5 was weakly expressed in glial fibrillary acidic protein-positive cells of the sham group; however, enhanced Cdk5 immunoreactivity was observed in the astrocytes of the $\mathrm{SAH}$ group, demonstrating that $\mathrm{Cdk} 5$ is activated in astrocytes following SAH (Fig. 2).

Roscovitine decreases Cdk5-pTyr15 expression. The $\mathrm{SAH}+$ roscovitine group exhibited significantly upregulated Cdk5-pTyr15 expression in the temporal cortex on day 1 after SAH compared with the sham + vehicle group $(\mathrm{P}<0.01$; Fig. 3$)$. Moreover, treatment with roscovitine suppressed Cdk5-pTyr15 expression in a dose-dependent manner in the $\mathrm{SAH}+$ roscovitine group, and treatment with high-dose roscovitine $(100 \mu \mathrm{g})$ significantly inhibited Cdk5-pTyr15 expression in the SAH + roscovitine group compared with the $\mathrm{SAH}+$ vehicle group $(\mathrm{P}<0.01$; Fig. 3).

Influence of roscovitine on brain edema and neurobehavioral deficits after $S A H$. SAH led to a significantly elevated level of brain water content in the $\mathrm{SAH}+$ vehicle group compared with 


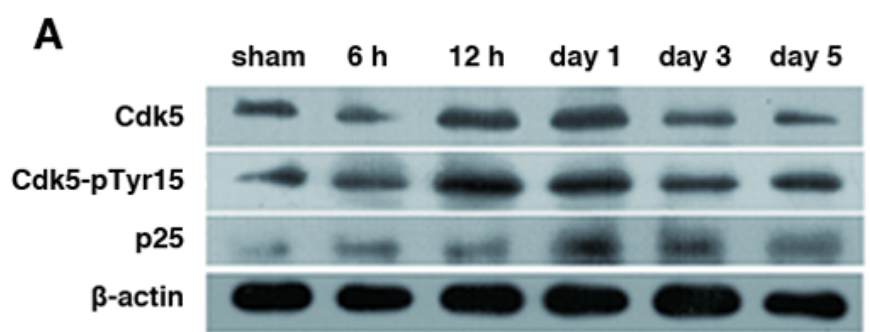

C

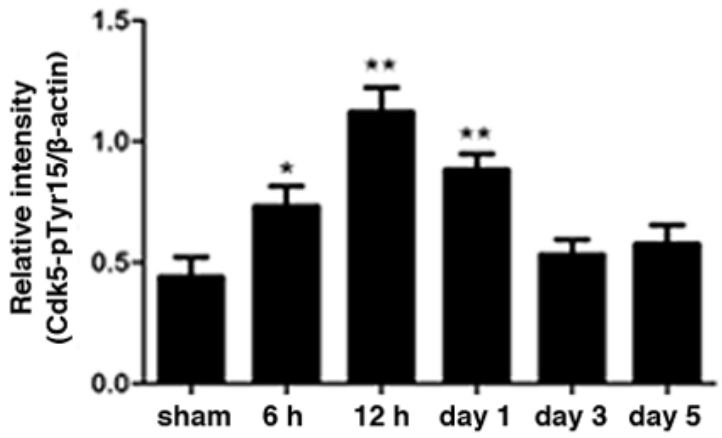

E

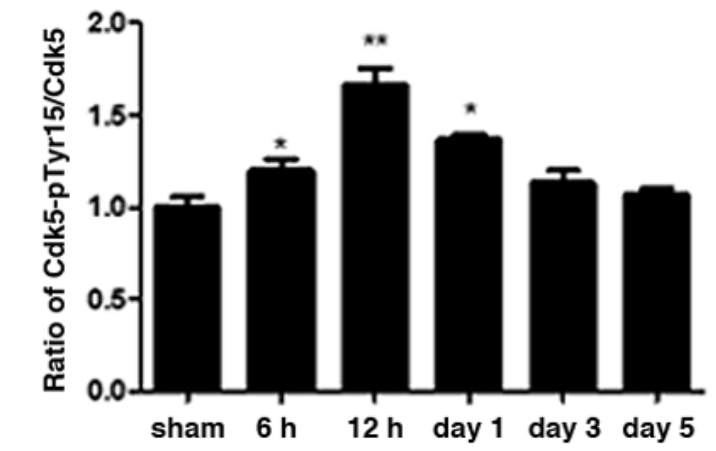

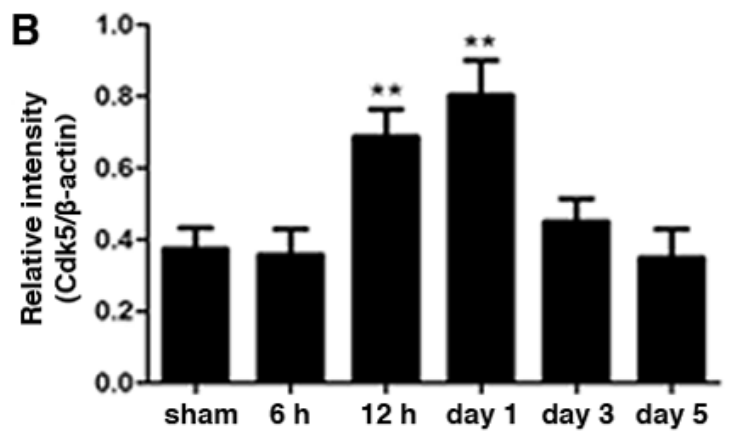

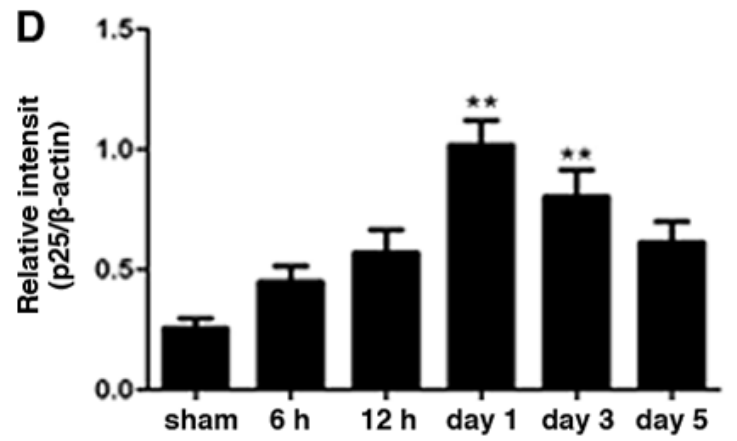

Figure 1. Expression levels of Cdk5, Cdk5-pTyr15 and p25 over time following SAH. (A) Representative autoradiograms of the expression levels of Cdk5, Cdk5-pTyr15 and p25 in the temporal cortex following SAH. Quantitative analysis of the western blotting results; (B) Cdk5 protein levels significantly increased at $12 \mathrm{~h}$ and on day 1 after SAH; (C) Cdk5-pTyr15 protein levels increased at 6 and $12 \mathrm{~h}$ and on day 1 after SAH; and (D) p25 protein levels increased on days 1 and 3 after SAH. (E) Quantified ratio of Cdk5-pTyr15/Cdk5 expression. ${ }^{*} \mathrm{P}<0.05,{ }^{* * *} \mathrm{P}<0.01$ compared with the sham group. Cdk5, cyclin-dependent kinase 5; Cdk5-pTyr15, Cdk5 phosphorylated at Tyr15; SAH, subarachnoid hemorrhage.

that in the sham + vehicle group. Roscovitine $(50 \mu \mathrm{g})$ administration failed to alleviate brain edema; however, roscovitine $(100 \mu \mathrm{g})$ administration elicited a noticeable decrease in brain water content in the $\mathrm{SAH}+$ roscovitine $(100 \mu \mathrm{g})$ group in contrast to the $\mathrm{SAH}+$ vehicle group $(\mathrm{P}<0.05$; Fig. $4 \mathrm{~A})$. These findings indicated that roscovitine attenuated brain edema.

The SAH + vehicle group demonstrated significantly lower neurological scores compared with the sham + vehicle group; however, the rats that received roscovitine $(100 \mu \mathrm{g})$ after $\mathrm{SAH}$ exhibited a significant increase in neurological scores compared with the $\mathrm{SAH}+$ vehicle group $(\mathrm{P}<0.05$; Fig. $4 \mathrm{~B})$. However, no significant difference was revealed in neurological behavior between the $\mathrm{SAH}+$ roscovitine $(50 \mu \mathrm{g})$ and $\mathrm{SAH}+$ vehicle groups.

Roscovitine-induced increases in neuronal survival after $S A H$. Nissl staining of the temporal cortex was performed to explore the neuroprotective effect of roscovitine. The sham + vehicle group exhibited neuronal cells with clear outline and compact structures and cytoplasm (Fig. 5A). After SAH, the majority of neurons were damaged and exhibited extensive degenerative changes. The neurons were sparsely arranged, appeared to have lost their integrity, and exhibited shrunken cytoplasm and swollen cell bodies (Fig. 5B-D). However, roscovitine $(100 \mu \mathrm{g})$ treatment significantly reduced the percentage of damaged neurons in the $\mathrm{SAH}+$ roscovitine $(100 \mu \mathrm{g})$ group compared with that in the $\mathrm{SAH}+$ vehicle group $(\mathrm{P}<0.05$; Fig. 5E).

SAH inhibits cortical neuronal apoptosis after SAH. The dose of $100 \mu \mathrm{g}$ of roscovitine was selected for this experiment because it was determined to be most effective dose based on the results of the above-described experiments. Few TUNEL-positive neurons were found in the temporal cortex of the sham + vehicle group (Fig. 6A-C). The $\mathrm{SAH}+$ vehicle group demonstrated a significantly increased percentage of apoptotic neurons compared with the sham + vehicle group; however, roscovitine treatment $(100 \mu \mathrm{g})$ significantly reduced the percentage of TUNEL-positive cells in the $\mathrm{SAH}+$ roscovitine $(100 \mu \mathrm{g})$ 


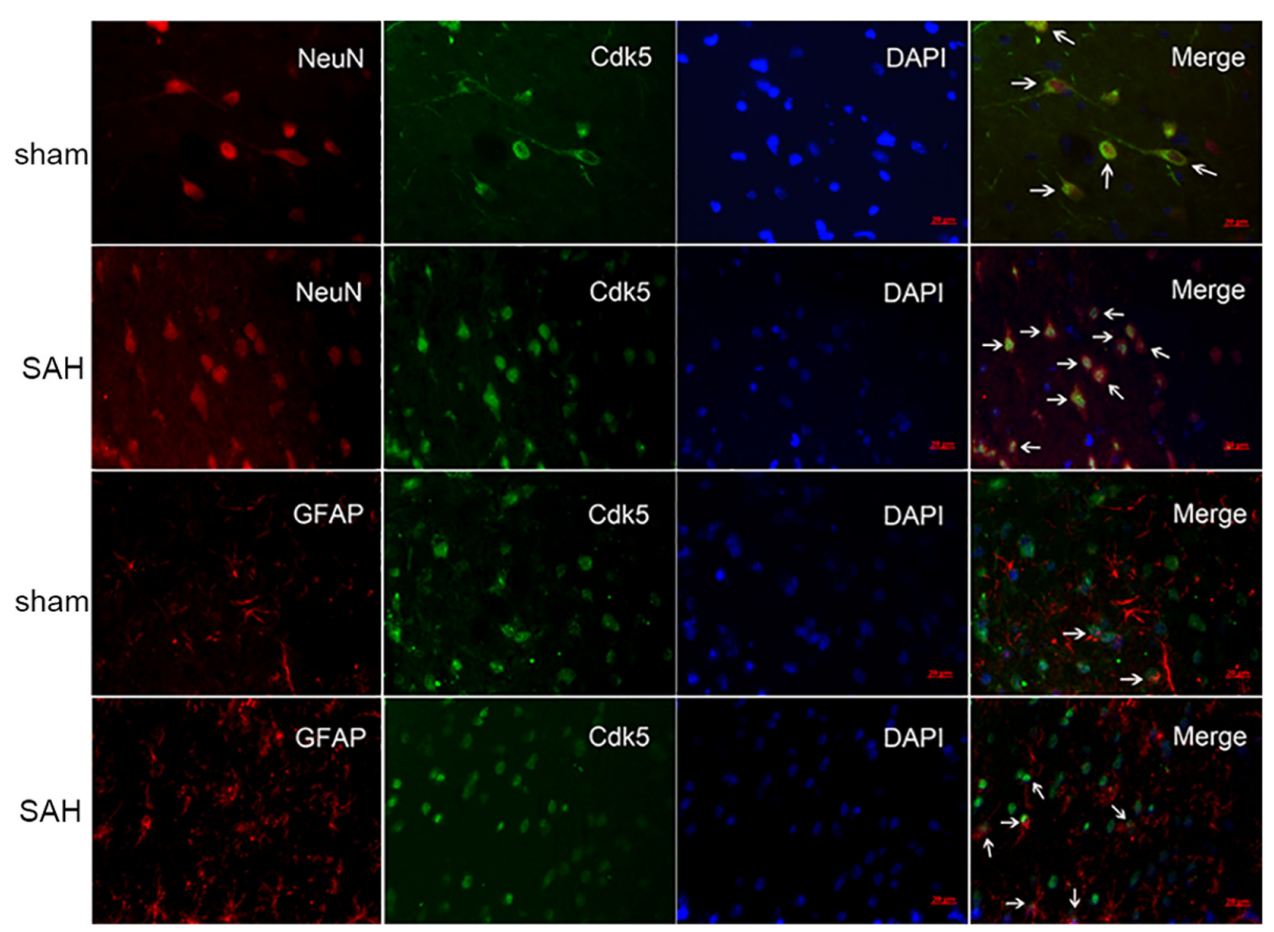

Figure 2. Double-immunofluorescence staining of Cdk5 after SAH. Cdk5 (green), NeuN (red) and GFAP (red) in the sham and day 1 post-SAH groups; nuclei were counterstained with DAPI (blue). Overlapping images show that Cdk5 was expressed in neurons and astrocytes in both the sham and SAH groups. Cdk5 was mainly expressed in the neuronal cytoplasm in the sham group; however, Cdk5 translocated to the nucleus after SAH (white arrows). Moreover, enhanced Cdk5 immunoreactivity was detected in the astrocytes of the SAH group compared with those of the sham group after SAH (scale bars, $20 \mu \mathrm{m}$ ). Cdk5, cyclin-dependent kinase 5; NeuN, neuronal nuclei; GFAP, glial fibrillary acidic protein; SAH, subarachnoid hemorrhage.

A
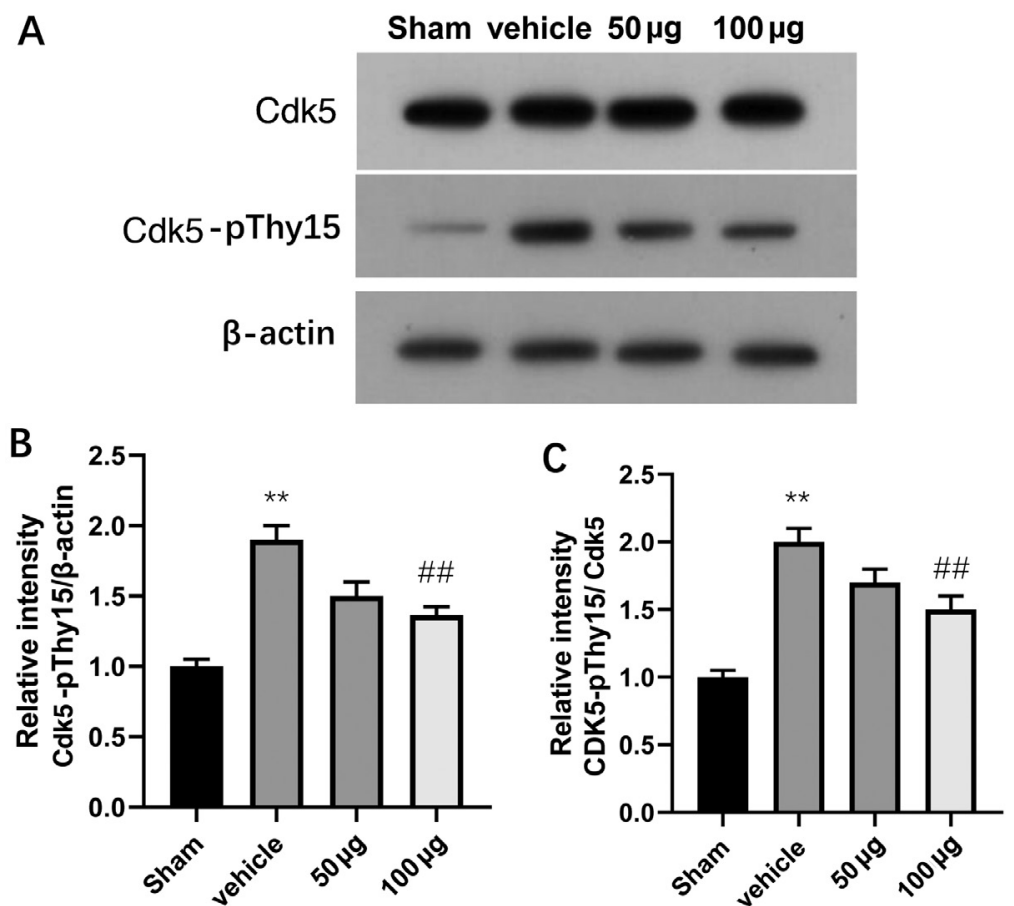

Figure 3. Effects of roscovitine on Cdk5-pTyr15 expression. (A) Cdk5-pTyr15 expression, as demonstrated by western blotting. (B) Quantitative western blotting results for Cdk5-pTyr15/ $\beta$-actin expression. (C) Quantitative western blotting results for Cdk5-pTyr15/Cdk5. Roscovitine $(100 \mu \mathrm{g})$ treatment significantly inhibited SAH-induced Cdk5-pTyr15 upregulation. ${ }^{* *} \mathrm{P}<0.01$ compared with the sham + vehicle group; ${ }^{\# \#} \mathrm{P}<0.01$ compared with the SAH + vehicle group. Cdk5-pTyr15. SAH, subarachnoid hemorrhage; Cdk5-pTyr15, Cdk5 phosphorylated at Tyr15.

group compared with the SAH + vehicle group (Fig. 6D). These results indicated that roscovitine treatment alleviated neuronal apoptosis after SAH.
Effects of roscovitine on the expression of cytochrome $c$ and caspase- 3 . Compared with the sham + vehicle group, the $\mathrm{SAH}+$ vehicle group displayed significantly increased 

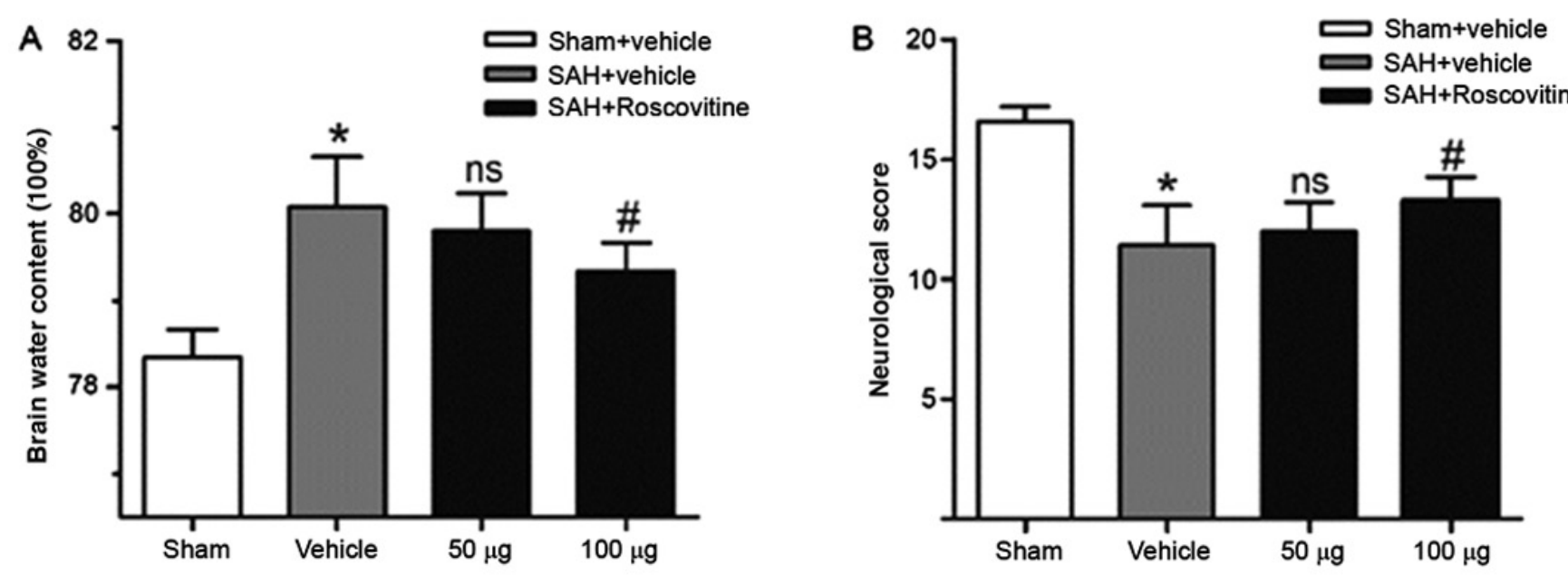

Figure 4. Effects of roscovitine on neurological scores and brain water content. (A) Brain water content was clearly elevated day 1 post after SAH. Roscovitine $(100 \mu \mathrm{g})$ administration noticeably decreased brain water content after SAH. (B) Neurological scores of the rats decreased on day 1 following SAH. Treatment with roscovitine $(100 \mu \mathrm{g})$ significantly improved neurological scores in the $\mathrm{SAH}+$ roscovitine group. $\mathrm{n}=6$ in each group. ${ }^{*} \mathrm{P}<0.05$ compared with the sham + vehicle group; ${ }^{\#} \mathrm{P}<0.05$ compared with the $\mathrm{SAH}+$ vehicle group; ${ }^{\text {ns }} \mathrm{P}>0.05$ compared with the $\mathrm{SAH}+$ vehicle group. $\mathrm{SAH}$, subarachnoid hemorrhage.

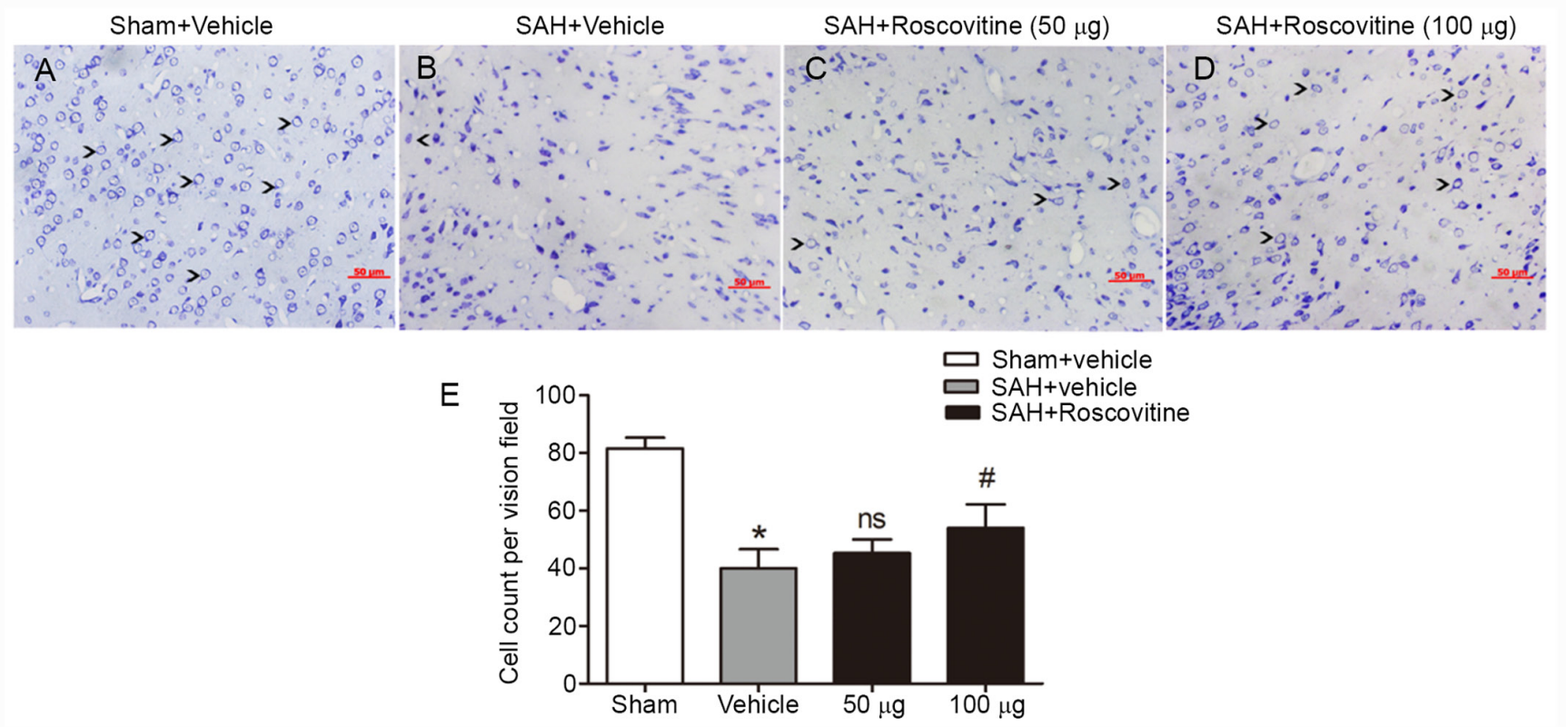

Figure 5. Effect of roscovitine on neuronal survival. Representative slides of Nissl staining in the temporal cortex of the (A) sham + vehicle, (B) SAH + vehicle, (C) $\mathrm{SAH}+$ roscovitine $(50 \mu \mathrm{g})$ and (D) SAH + roscovitine $(100 \mu \mathrm{g})$ groups. (E) SAH + vehicle group exhibited severe neuronal loss; however, roscovitine $(100 \mu \mathrm{g})$ significantly increased the percentage of surviving neurons. $\mathrm{n}=6$ in each group. Scale bars, $50 \mu \mathrm{m}$. " $\mathrm{P}<0.05$ compared with the sham + vehicle group; ${ }^{\text {" }} \mathrm{P}<0.05$ compared with the $\mathrm{SAH}+$ vehicle group; ${ }^{\text {ns }} \mathrm{P}>0.05$ compared with the $\mathrm{SAH}+$ vehicle group. $\mathrm{SAH}$, subarachnoid hemorrhage.

expression levels of cytochrome $\mathrm{c}$ and caspase-3 $(\mathrm{P}<0.05$; Fig. 7A and B). However, cytochrome release and caspase-3 cleavage were both significantly decreased after roscovitine $(100 \mu \mathrm{g})$ treatment in the $\mathrm{SAH}+$ roscovitine $(100 \mu \mathrm{g})$ group compared with the SAH + vehicle group (Fig. 7A and B).

\section{Discussion}

The present study determined that SAH led to upregulation of the expression levels of Cdk5, p25 and Cdk5-pTyr15 proteins. Immunofluorescence staining highlighted the expression of Cdk5 after SAH and that Cdk5 underwent nuclear translocation in neurons. In addition, intracisternal administration of roscovitine effectively improved neurological function, reduced the number of apoptotic cells and alleviated brain edema. Furthermore, SAH induced the upregulation of the expression levels of cytochrome c and caspase-3, whereas roscovitine treatment significantly reduced the expression of these proteins. Thus, the present study provided strong evidence that Cdk5 was an important participant in the development of EBI following SAH and may represent a focus for potential SAH treatment in the future.

Previous studies confirmed that $\mathrm{Cdk} 5$ is activated in several diseases of the nervous system, such as Alzheimer's disease (30), cerebral ischemia (31) and Parkinson's disease (32). One regulatory mechanism of $\mathrm{Cdk} 5$ kinase activity is the 


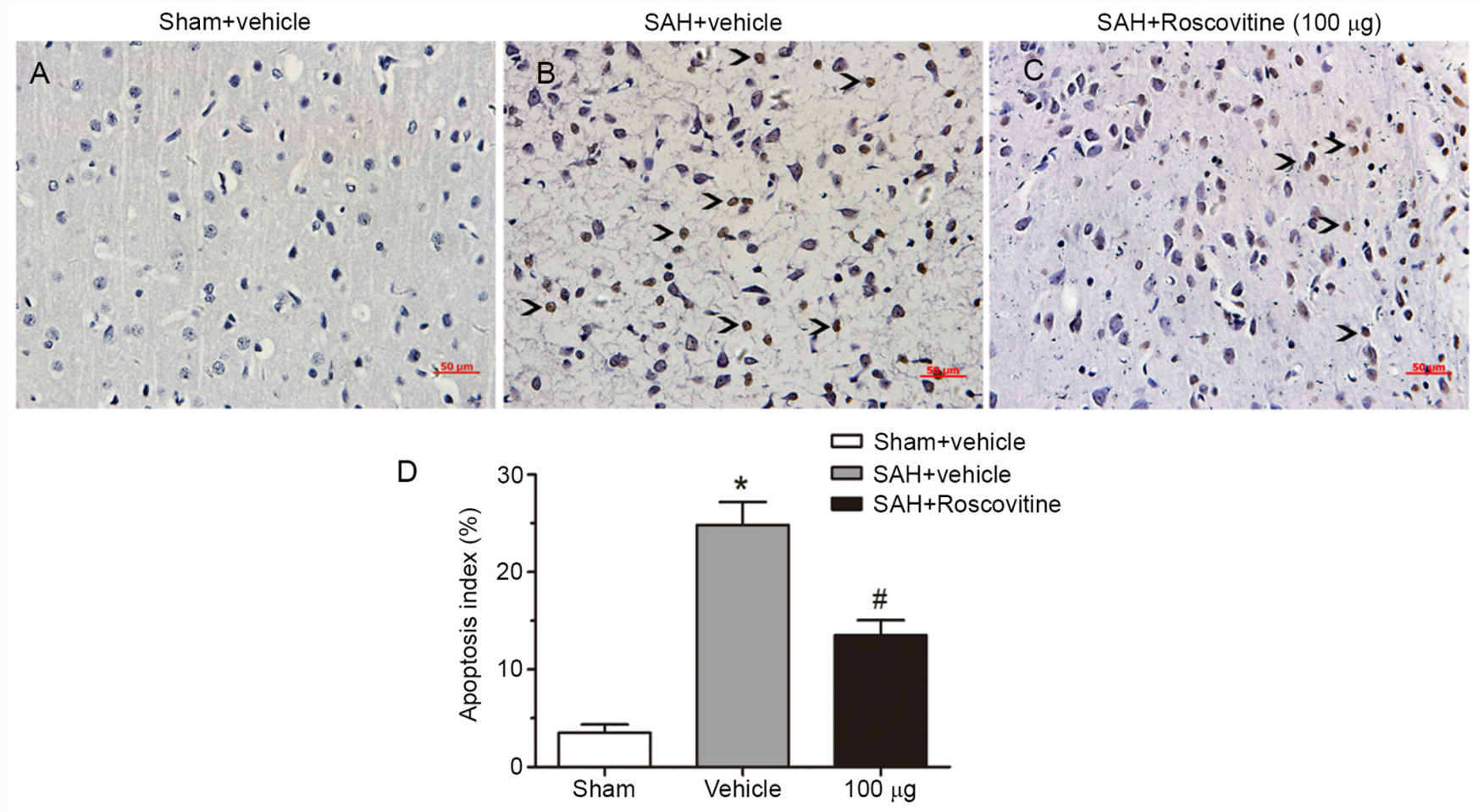

Figure 6. TUNEL staining results. (A) Sham + vehicle, (B) SAH + vehicle, and (C) SAH + roscovitine (100 $\mu \mathrm{g})$ groups. (D) Apoptotic index (percentage of apoptosis) was significantly greater in the $\mathrm{SAH}+$ vehicle group compared with the sham + vehicle group. Roscovitine treatment significantly decreased the post-SAH apoptotic index. Scale bars, $50 \mu \mathrm{m}$. ${ }^{*} \mathrm{P}<0.05$ compared with the sham + vehicle group; ${ }^{*} \mathrm{P}<0.05$ compared with the $\mathrm{SAH}+$ vehicle group. SAH, subarachnoid hemorrhage.

A

cytochrome c

$\beta$-actin

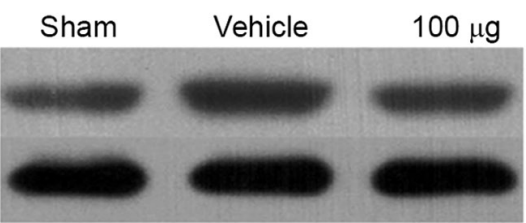

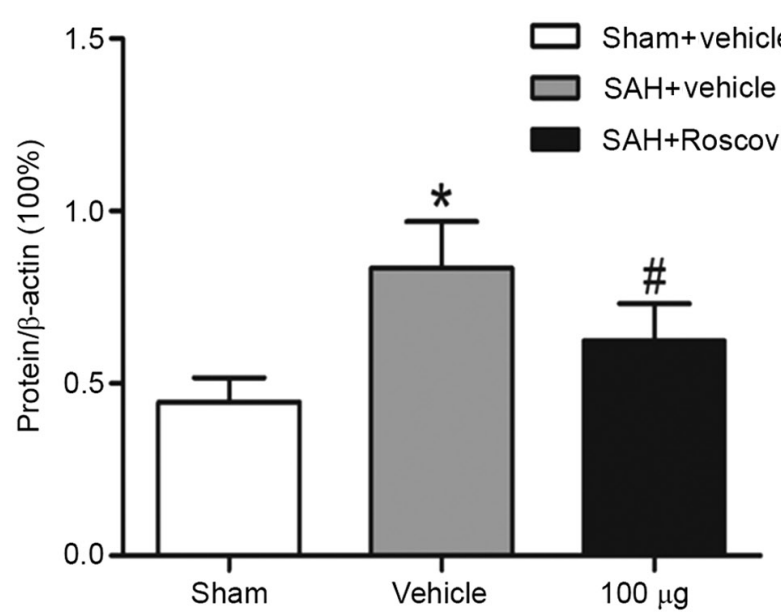

B

Caspase-3

$\beta$-actin
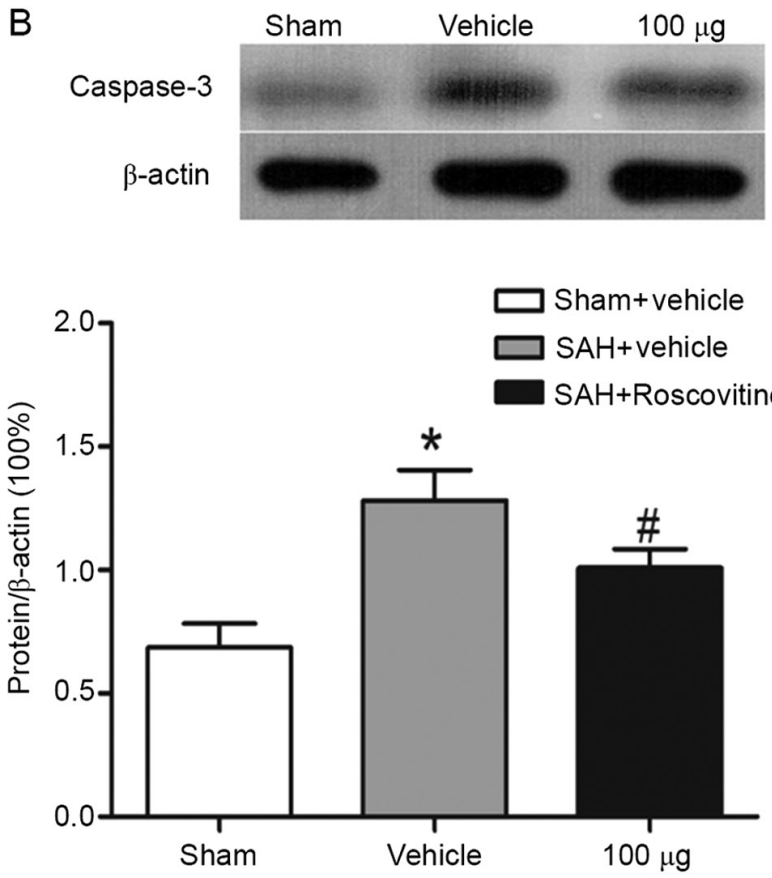

Figure 7. Western blotting results. (A) Cytochrome $c$ and (B) cleaved caspase-3 in the post-SAH temporal cortex. Expression of cytochrome $c$ and caspase-3 increased significantly on day 1 after SAH, and treatment with roscovitine $(100 \mu \mathrm{g})$ significantly reduced the expression of these proteins. ${ }^{*} \mathrm{P}<0.05$ compared with the sham + vehicle group; ${ }^{\#} \mathrm{P}<0.05$ compared with the $\mathrm{SAH}+$ vehicle group. $\mathrm{SAH}$, subarachnoid hemorrhage.

phosphorylation of conserved residues (33). Phosphorylation of Tyr15 residue of Cdk5 has a stimulatory effect and increases Cdk5 kinase activity $(14,34)$. In the present study, Cdk5 and Cdk5-pTyr15 were significantly upregulated in the rat cortex following $\mathrm{SAH}$, indicating that Cdk5 kinase activity was increased. In addition, as aberrant activation of Cdk5 requires the help of the activator protein p25 (35), the protein levels of p25 were investigated and it was revealed that p25 expression 
was elevated after SAH. p25 has a significantly longer half-life compared with other proteins and can have deleterious effects in neurons $(30,36)$. Increases in p25 protein expression can prolong Cdk5 activation. The current study provided evidence of $\mathrm{Cdk} 5$ activation in the rat brain after $\mathrm{SAH}$.

A previous study suggested that $\mathrm{Cdk} 5$ is a regulator of various neuronal cell death cascades (37). However, some studies have provided contradictory evidence indicating that Cdk5 provides neuroprotection in some conditions $(38,39)$. Several studies have revealed that the influence of Cdk5 on cell death depended on its subcellular localization as opposed to its activity. These studies suggested that $\mathrm{Cdk} 5$ plays a pro-survival role in the cytoplasm but participates in death signaling within the nucleus (40-42). The present study suggested that Cdk5 was mostly expressed in the cytoplasm in the sham group but translocated to the nucleus after $\mathrm{SAH}$, indicating that $\mathrm{Cdk} 5$ participates in neuronal cell death after SAH.

Brain edema has been described as an accurate predictor of EBI and an independent risk factor for unsatisfactory outcomes in patients with SAH (43). Edema increases the mass and ICP following SAH, which may lead to direct brain tissue damage and ultimately, herniation. Mounting evidence and data has demonstrated that apoptosis is strongly associated with the development of brain edema after SAH $(44,45)$. Endothelial cell apoptosis causes BBB breakdown, thereby inducing brain edema. Moreover, neuronal apoptosis contributes to cytotoxic brain edema, resulting in subsequent neurological deficits $(46,47)$. To further elucidate the effects of $\mathrm{Cdk} 5$, the present study treated SAH rats with roscovitine, a selective inhibitor of Cdk5. As expected, roscovitine significantly alleviated brain edema after SAH. Furthermore, roscovitine also reduced the number of apoptotic cells and accelerated the recovery of neurological function following $\mathrm{SAH}$. Therefore, it is concluded that the inhibition of Cdk5 alleviated brain edema and decreased neurobehavioral deficits by mitigating neuronal apoptosis following SAH.

Accumulating evidence indicates that apoptosis is an important intracellular pathway following SAH $(48,49)$. Although the precise mechanisms underlying apoptosis have not been fully elucidated, mitochondrial dysfunction has been demonstrated to be involved in apoptosis (40). During apoptosis, the permeability of the outer mitochondrial membrane increases, leading to the leakage of cytochrome $\mathrm{c}$ into the cytosol. Interaction between cytochrome $\mathrm{c}$ and Apaf-1 facilitates the formation of an apoptosome and activates caspase-3, leading to neuronal apoptosis (50). Cdk5 is involved in mitochondrial dysfunction; it triggers mitochondrial fission and is an upstream regulator of the mitochondrial pathway (51). Blocking Cdk5 activity prevents mitochondria from releasing cytochrome $\mathrm{c}$ and preserves mitochondrial integrity in injured neurons (52). The current study measured cytochrome $\mathrm{c}$ and caspase-3 protein levels to further explore the mechanism by which roscovitine exerts its anti-apoptotic effects. The results of the present study suggested that the expression of cytochrome c and caspase-3 substantially increased after SAH, which confirmed the results of previous studies. However, treatment with roscovitine $(100 \mu \mathrm{g})$ significantly decreased the expression levels of cytochrome $\mathrm{c}$ and caspase-3, indicating that roscovitine attenuates neuronal apoptosis after SAH by inhibiting the expression levels of cytochrome c and caspase-3, thereby reducing brain edema and restoring neurological function.

The present study has certain limitations. Roscovitine is not a specific inhibitor of $\mathrm{Cdk} 5$ because it also inhibits $\mathrm{Cdc} 2$ and Cdk2 (53). However, previous studies have revealed that Cdk5 is expressed mainly in post-mitotic neurons, whereas Cdc2 and $\mathrm{Cdk} 2$ are expressed only at the embryonic stage $(54,55)$. Furthermore, all Cdk activity other than Cdk5 activity is suppressed in mature neurons $(56,57)$. Kinase activity cannot be directly measured, so the content of phosporylated-cdk5 was indirectly measured based on previous literature (58). Therefore, in conclusion, it is reasonable to speculate that the neuroprotective effects noted in this experiment occurred mainly through the inhibition of $\mathrm{Cdk} 5$ by roscovitine.

In summary, the results of the present study demonstrated that SAH significantly upregulated the expression levels of Cdk5, p25 and Cdk5-pTyr15 and induced apparent EBI. Cdk5 inhibition by roscovitine protected neurons from apoptosis following SAH. Moreover, caspase-3 and cytochrome c were closely associated with the anti-apoptotic effects of roscovitine on the brain cells following experimental SAH. These data and results demonstrated that Cdk5 plays a role in EBI after SAH and provides support for the hypothesis that Cdk5 inhibitors can be developed as therapeutic agents for $\mathrm{SAH}$.

\section{Acknowledgements}

The authors would like to thank Dr Hua Li, Dr Chun-Xi Wang and Dr Xiao-Ming Zhou from the Affiliated Suqian Hospital of Xuzhou Medical University (Suqian, China) for providing suggestions. In addition, the authors would like to acknowledge the assistance provided by the following three pathologists: Dr Qing-Song Wang, Dr Hai-Feng Liu and Dr Peng Zhao, Department of Pathology of the Affiliated Suqian Hospital of Xuzhou Medical University (Suqian, China).

\section{Funding}

This work was supported by The National Natural Science Foundation of China (grant no. 81070974), The Jiangsu Provincial Key Subject (grant no. X4200722) and The Jinling Hospital of Nanjing, China (grant no. 2010Q017).

\section{Availability of data and materials}

The datasets used and/or analyzed during the current study are available from the corresponding author on reasonable request.

\section{Authors' contributions}

YD, LZ and JZ conceived and designed the project. LZ and JZ confirm the authenticity of all the raw data and actually performed the experiments. WZ, HL, XG and JLi contributed to data/evidence collection and analysis. YD, LZ, JLiu, XN and $\mathrm{JZ}$ analyzed and interpreted the data. JZ wrote the manuscript. All authors have read and approved the final manuscript. 


\section{Ethics approval and consent to participate}

All study protocols regarding surgical procedures and animal usage were approved by The Ethics Committee of the Affiliated Suqian Hospital of Xuzhou Medical University (Suqian, China).

\section{Patient consent for publication}

Not applicable.

\section{Competing interests}

The authors declare that they have no competing interests.

\section{References}

1. Connolly ES Jr, Rabinstein AA, Carhuapoma JR, Derdeyn CP, Dion J, Higashida RT, Hoh BL, Kirkness CJ, Naidech AM, Ogilvy CS, et al: Guidelines for the management of aneurysmal subarachnoid hemorrhage: A guideline for healthcare professionals from the American heart association/american stroke association. Stroke 43: 1711-1737, 2012.

2. Hop JW, Rinkel GJ, Algra A and van Gijn J: Case-fatality rates and functional outcome after subarachnoid hemorrhage: A systematic review. Stroke 28: 660-664, 1997.

3. Mackey J, Khoury JC, Alwell K, Moomaw CJ, Kissela BM, Flaherty ML, Adeoye O, Woo D, Ferioli S, De Los Rios La Rosa F, et al: Stable incidence but declining case-fatality rates of subarachnoid hemorrhage in a population. Neurology 87 2192-2197, 2016

4. Macdonald RL, Kassell NF, Mayer S, Ruefenacht D, Schmiedek P, Weidauer S, Frey A, Roux S and Pasqualin A; CONSCIOUS-1 Investigators: Clazosentan to overcome neurological ischemia and infarction occurring after subarachnoid hemorrhage (CONSCIOUS-1): Randomized, double-blind, placebo-controlled phase 2 dose-finding trial. Stroke 39 3015-3021, 2008.

5. Sehba FA, Hou J, Pluta RM and Zhang JH: The importance of early brain injury after subarachnoid hemorrhage. Prog Neurobiol 97: 14-37, 2012.

6. Chaudhry SR, Kahlert UD, Kinfe TM, Endl E, Dolf A, Niemelä M, Hänggi D and Muhammad S: Differential polarization and activation dynamics of systemic T helper cell subsets after aneurysmal subarachnoid hemorrhage $(\mathrm{SAH})$ and during post-SAH complications. Sci Rep 11: 14226, 2021.

7. Liu L, Kawakita F, Fujimoto M, Nakano F, Imanaka-Yoshida K, Yoshida T and Suzuki H: Role of periostin in early brain injury after subarachnoid hemorrhage in mice. Stroke 48: 1108-1111, 2017. Suzuki H, Ayer R, Sugawara T, Chen W, Sozen T, Hasegawa Y, Kanamaru K and Zhang JH: Protective effects of recombinant osteopontin on early brain injury after subarachnoid hemorrhage in rats. Crit Care Med 38: 612-618, 2010.

8. Uekawa K, Hasegawa Y, Ma M, Nakagawa T, Katayama T, Sueta D, Toyama K, Kataoka K, Koibuchi N, Kawano T, et al: Rosuvastatin ameliorates early brain injury after subarachnoid hemorrhage via suppression of superoxide formation and nuclear factor-kappa B activation in rats. J Stroke Cerebrovasc Dis 23: 1429-1439, 2014

9. Feng D, Wang W, Dong Y, Wu L, Huang J, Ma Y, Zhang Z, Wu S, Gao $\mathrm{G}$ and Qin H: Ceftriaxone alleviates early brain injury after subarachnoid hemorrhage by increasing excitatory amino acid transporter 2 expression via the $\mathrm{PI} 3 \mathrm{~K} / \mathrm{Akt} / \mathrm{NF}-\mathrm{\kappa B}$ signaling pathway. Neuroscience 268: 21-32, 2014.

10. Wang Z, Guo S, Wang J, Shen Y, Zhang J and Wu Q: Nrf2/HO-1 mediates the neuroprotective effect of mangiferin on early brain injury after subarachnoid hemorrhage by attenuating mitochondria-related apoptosis and neuroinflammation. Sci Rep 7: 11883, 2017.

11. Mo J, Enkhjargal B, Travis ZD, Zhou K, Wu P, Zhang G, Zhu Q, Zhang T, Peng J, Xu W, et al: AVE 0991 attenuates oxidative stress and neuronal apoptosis via Mas/PKA/CREB/UCP-2 pathway after subarachnoid hemorrhage in rats. Redox Biol 20: $75-86,2019$.
12. Pang J, Chen Y, Kuai L, Yang P, Peng J, Wu Y, Chen Y, Vitek MP, Chen L, Sun X and Jiang Y: Inhibition of blood-brain barrier disruption by an apolipoprotein E-mimetic peptide ameliorates early brain injury in experimental subarachnoid hemorrhage. Transl Stroke Res 8: 257-272, 2017.

13. Mangold N, Pippin J, Unnersjoe-Jess D, Koehler S, Shankland S, Brähler S, Schermer B, Benzing T, Brinkkoetter PT and Hagmann H: The atypical cyclin-dependent kinase 5 (Cdk5) guards podocytes from apoptosis in glomerular disease while being dispensable for podocyte development. Cells 10: 2464, 2021.

14. Roufayel R and Murshid N: CDK5: Key regulator of apoptosis and cell survival. Biomedicines 7: 88, 2019.

15. Shah K and Lahiri DK: A tale of the good and bad: Remodeling of the microtubule network in the brain by Cdk5. Mol Neurobiol 54: 2255-2268, 2017.

16. Sharma $S$ and Sicinski P. A kinase of many talents: Non-neuronal functions of CDK5 in development and disease. Open Biol 10: 190287, 2020.

17. Mushtaq G, Greig NH, Anwar F, Al-Abbasi FA, Zamzami MA, Al-Talhi HA and Kamal MA: Neuroprotective mechanisms mediated by CDK5 inhibition. Curr Pharm Des 22: 527-534, 2016.

18. Menn B, Bach S, Blevins TL, Campbell M, Meijer L and Timsit S: Delayed treatment with systemic (S)-roscovitine provides neuroprotection and inhibits in vivo CDK5 activity increase in animal stroke models. PLoS One 5: e12117, 2010.

19. Tuo QZ, Liuyang ZY, Lei P, Yan X, Shentu YP, Liang JW, Zhou H, Pei L, Xiong Y, Hou TY, et al: Zinc induces CDK5 activation and neuronal death through CDK5-Tyr15 phosphorylation in ischemic stroke. Cell Death Dis 9: 870, 2018.

20. Zhou YF, Wang J, Deng MF, Chi B, Wei N, Chen JG, Liu D, Yin X, Lu Y and Zhu LQ: The peptide-directed lysosomal degradation of CDK5 exerts therapeutic effects against stroke. Aging Dis 10: $1140-1145,2019$.

21. Kumar S, Rajput MK and Tickoo SB: Laws, regulations, policies and guidelines governing the care and use of laboratory animals. Essentials of laboratory animal science: Principles and practices. Springer, Singapore, pp23-38, 2021.

22. Sun Q, Dai Y, Zhang X, Hu YC, Zhang D, Li W, Zhang XS, Zhu JH, Zhou ML and Hang CH: Expression and cell distribution of myeloid differentiation primary response protein 88 in the cerebral cortex following experimental subarachnoid hemorrhage in rats: A pilot study. Brain Res 1520: 134-144, 2013.

23. Wang KC, Tang SC, Lee JE, Tsai JC, Lai DM, Lin WC, Lin CP Tu YK and Hsieh ST: Impaired microcirculation after subarachnoid hemorrhage in an in vivo animal model. Sci Rep 8: 13315, 2018.

24. Li R, Liu W, Yin J, Chen Y, Guo S, Fan H, Li X, Zhang X, He X and Duan C: TSG-6 attenuates inflammation-induced brain injury via modulation of microglial polarization in $\mathrm{SAH}$ rats through the SOCS3/STAT3 pathway. J Neuroinflammation 15: $231,2018$.

25. Lee JY, Sagher O, Keep R, Hua Y and Xi G: Comparison of experimental rat models of early brain injury after subarachnoid hemorrhage. Neurosurgery 65: 331-343, 2009.

26. Hilton GD, Stoica BA, Byrnes KR and Faden AI: Roscovitine reduces neuronal loss, glial activation, and neurologic deficits after brain trauma. J Cereb Blood Flow Metab 28: 1845-1859, 2008.

27. Nicolás P, Lassalle VL and Ferreira ML: Quantification of immobilized Candida antarctica lipase B (CALB) using ICP-AES combined with Bradford method. Enzyme Microb Technol 97: 97-103, 2017.

28. Zhuang Z, Zhou ML, You WC, Zhu L, Ma CY, Sun XJ and Shi JX: Hydrogen-rich saline alleviates early brain injury via reducing oxidative stress and brain edema following experimental subarachnoid hemorrhage in rabbits. BMC Neurosci 13: 47, 2012.

29. Garcia JH, Wagner S, Liu KF and Hu XJ: Neurological deficit and extent of neuronal necrosis attributable to middle cerebral artery occlusion in rats. Statistical validation. Stroke 26: 627-635, 1995.

30. Liu X, Feng Z, Du L, Huang Y, Ge J, Deng Y and Mei Z: The potential role of MicroRNA-124 in cerebral ischemia injury. Int J Mol Sci 21: 120, 2019.

31. Cheng X, Xu S, Zhang C, Qin K, Yan J and Shao X: The BRCC3 regulated by $\mathrm{Cdk} 5$ promotes the activation of neuronal NLRP3 inflammasome in Parkinson's disease models. Biochem Biophys Res Commun 522: 647-654, 2020. 
32. Ji YB, Zhuang PP, Ji Z, Wu YM, Gu Y, Gao XY, Pan SY and Hu YF: TFP5 peptide, derived from CDK5-activating cofactor p35, provides neuroprotection in early-stage of adult ischemic stroke. Sci Rep 7: 40013, 2017.

33. Shupp A, Casimiro MC and Pestell RG: Biological functions of CDK5 and potential CDK5 targeted clinical treatments. Oncotarget 8: 17373-17382, 2017.

34. Zhang B, Tan VB, Lim KM and Tay TE: The activation and inhibition of cyclin-dependent kinase- 5 by phosphorylation. Biochemistry 46: 10841-10851, 2007.

35. Ko YU, Kim C, Lee J, Kim D, Kim Y, Yun N and Oh YJ: Site-specific phosphorylation of Fbxw 7 by $\mathrm{Cdk} 5 / \mathrm{p} 25$ and its resulting decreased stability are linked to glutamate-induced excitotoxicity. Cell Death Dis 10: 579, 2019.

36. Lee MS, Kwon YT, Li M, Peng J, Friedlander RM and Tsai LH: Neurotoxicity induces cleavage of p35 to p25 by calpain. Nature 405: 360-364, 2000.

37. Zhang H, Chang L, Zhang H,Nie J, ZhangZ, Yang X, Vuong AM, Wang Z, Chen A and Niu Q: Calpain-2/p35-p25/Cdk5 pathway is involved in the neuronal apoptosis induced by polybrominated diphenyl ether-153. Toxicol Lett 277: 41-53, 2017.

38. Tucker D, Lu Y and Zhang Q: From mitochondrial function to neuroprotection-an emerging role for methylene blue. Mol Neurobiol 55: 5137-5153, 2018

39. Sénécal V, Barat C and Tremblay MJ: The delicate balance between neurotoxicity and neuroprotection in the context of HIV-1 infection. Glia 69: 255-280, 2021.

40. Tiernan CT, Ginsberg SD, He B, Ward SM, GuillozetBongaarts AL, Kanaan NM, Mufson EJ and Counts SE: Pretangle pathology within cholinergic nucleus basalis neurons coincides with neurotrophic and neurotransmitter receptor gene dysregulation during the progression of Alzheimer's disease. Neurobiol Dis 117: 125-136, 2018

41. Sun YQ, Xie JW, Xie HT, Chen PC, Zhang XL, Zheng $\mathrm{CH}$ Li P, Wang JB, Lin JX, Cao LL, et al: Expression of CRM1 and CDK 5 shows high prognostic accuracy for gastric cancer. World J Gastroenterol 23: 2012-2022, 2017.

42. Ruegsegger GN, Toedebusch RG, Childs TE, Grigsby KB and Booth FW: Loss of Cdk5 function in the nucleus accumbens decreases wheel running and may mediate age-related declines in voluntary physical activity. J Physiol 595: 363-384, 2017.

43. Tanabe J, Nakahara I, Matsumoto S, Suyama Y, Morioka J, Oda J, Hasebe A, Suzuki T, Watanabe S, Suyama K, et al: Cortical blood flow insufficiency scores with computed tomography perfusion can predict outcomes in aneurysmal subarachnoid hemorrhage patients: A cohort study. Neurocrit Care 34: 946-955, 2021.

44. Qian C, Jin J, Chen J, Li J, Yu X, Mo H and Chen G: SIRT1 activation by resveratrol reduces brain edema and neuronal apoptosis in an experimental rat subarachnoid hemorrhage model. Mol Med Rep 16: 9627-9635, 2017.

45. Shamsi Meymandi M, Soltani Z, Sepehri G, Amiresmaili S, Farahani F and Moeini Aghtaei M: Effects of pregabalin on brain edema, neurologic and histologic outcomes in experimental traumatic brain injury. Brain Res Bull 140: 169-175, 2018
46. Liu XC, Wu CZ, Hu XF, Wang TL, Jin XP, Ke SF, Wang E and Wu G: Gastrodin attenuates neuronal apoptosis and neurological deficits after experimental intracerebral hemorrhage. J Stroke Cerebrovasc Dis 29: 104483, 2020.

47. Sadeghian N, Shadman J, Moradi A, Ghasem Golmohammadi M and Panahpour $\mathrm{H}$ : Calcitriol protects the blood-brain barrier integrity against ischemic stroke and reduces vasogenic brain edema via antioxidant and antiapoptotic actions in rats. Brain Res Bull 150: 281-289, 2019.

48. Sun J and Nan G: The extracellular signal-regulated kinase $1 / 2$ pathway in neurological diseases: A potential therapeutic target (review). Int J Mol Med 39: 1338-1346, 2017.

49. Huang YH, Chung CL, Tsai HP, Tzou RD, Wu SC, Chai CY, Lee TC and Kwan AL: Impact of hyperglycemia on neuronal apoptosis after subarachnoid hemorrhage in rodent brain: An experimental research. Int J Surg 83: 246-252, 2020.

50. Yadav N, Gogada R, O'Malley J, Gundampati RK, Jayanthi S, Hashmi S, Lella R, Zhang D, Wang J, Kumar R, et al: Molecular insights on cytochrome $\mathrm{c}$ and nucleotide regulation of apoptosome function and its implication in cancer. Biochim Biophys Acta Mol Cell Res 1867: 118573, 2020.

51. Guo MY, Shang L, Hu YY, Jiang LP, Wan YY, Zhou QQ, Zhang K, Liao HF, Yi JL and Han XJ: The role of Cdk5-mediated Drp1 phosphorylation in $\mathrm{A} \beta_{1-42}$ induced mitochondrial fission and neuronal apoptosis. J Cell Biochem 119: 4815-4825, 2018.

52. Seager R, Lee L, Henley JM and Wilkinson KA: Mechanisms and roles of mitochondrial localisation and dynamics in neuronal function. Neuronal Signal 4: NS20200008, 2020.

53. Kim JE, Park H, Choi SH, Kong MJ and Kang TC: Roscovitine attenuates microglia activation and monocyte infiltration via $\mathrm{p} 38$ MAPK inhibition in the rat frontoparietal cortex following status epilepticus. Cells 8: 746, 2019.

54. Kartha CC: Cell cycle regulation in cardiomyocytes. In: Cardiomyocytes in health and disease. Springer, Cham, pp25-39, 2021.

55. Chao AC, Chen $\mathrm{CH}, \mathrm{Wu} \mathrm{MH}$, Hou BY and Yang DI: Roles of Id1/HIF-1 and CDK5/HIF-1 in cell cycle reentry induced by amyloid-beta peptide in post-mitotic cortical neuron. Biochim Biophys Acta Mol Cell Res 1867: 118628, 2020.

56. Cortés N, Guzmán-Martínez L, Andrade V, González A and Maccioni RB: CDK5: A unique CDK and its multiple roles in the nervous system. J Alzheimers Dis 68: 843-855, 2019.

57. Chen SD, Yang JL, Lin YC, Chao AC and Yang DI: Emerging roles of inhibitor of differentiation-1 in Alzheimer's disease: Cell cycle reentry and beyond. Cells 9: 1746, 2020.

58. Lee J, Ko YU, Chung Y, Yun N, Kim M, Kim K and Oh YJ: The acetylation of cyclin-dependent kinase 5 at lysine 33 regulates kinase activity and neurite length in hippocampal neurons. Sci Rep 8: 13676, 2018.

This work is licensed under a Creative Commons Attribution-NonCommercial-NoDerivatives 4.0 International (CC BY-NC-ND 4.0) License. 